\title{
Research on an Economic Localization Approach
}

\author{
Fengchun Yin $^{1} \&$ Jun Yin ${ }^{2}$ \\ ${ }^{1}$ Yancheng Teachers University, Yancheng, China \\ ${ }^{2}$ Yancheng Senior Experiment High School, Yancheng, China \\ Correspondence: Fengchun Yin, Yancheng Teachers University, Yancheng, China. E-mail: yinfc@yctu.edu.cn \\ Received: Dec. 5, 2018 \\ Accepted: Jan. 12, 2019 \\ Online Published: January 18, 2019 \\ doi:10.5539/cis. v12n1p44 \\ URL: https://doi.org/10.5539/cis.v12n1p44
}

\begin{abstract}
With the development of wireless network and the wide application of pervasive computing technology, the location-based service (LBS) needs more and more location information for mobile users. At present, the outdoor positioning system based on satellite signals has been very mature, but it can not be applied in the complex indoor environment. Therefore, indoor positioning technology has rapidly become a research hotspot. At the same time, the rapid development of wireless network technology, because of its fast communication speed, easy deployment and other characteristics, WiFi-based indoor positioning technology has been widely concerned and studied. Therefore, this paper takes an economic WiFi-based indoor positioning method as the research foundation, and studies the corresponding improved algorithm aiming at the existing problems.
\end{abstract}

Keywords: Locailization, WiFi, RSSI, Fingerprint

\section{Introduction}

In recent years, the wireless positioning system has developed rapidly, especially in the application of mobile devices. From automobile navigation to mobile tracking and positioning, it can be said that it is everywhere. It has penetrated into people's lives. People can query all kinds of location information at any time to ensure the safety and convenience of people's travel. It has brought great benefits to people's lives.

At present, many wireless technologies are applied to indoor positioning, such as A-GPS, infrared, UWB, RFID radio frequency tag, Zigbee, WSN wireless sensor network technology and WLAN, which are based on a special signal to measure and calculate, and then according to the corresponding positioning algorithm to estimate the object to be measured. Compared with other indoor wireless positioning technologies, WiFi has its unique advantages and is a hot research topic of indoor positioning technology. WiFi hotspots are all over every corner of the city and buildings. The concept of wireless city emerges as the times require. Its ubiquity makes the indoor positioning system based on WiFi cheap and easy to install hardware. It is a good complement to GPS positioning system or cellular positioning system. With the wide use of various wireless mobile devices, especially smart phones, WiFi positioning provides a good market, making the rapid development of WiFi positioning technology, occupying the main market of indoor positioning.

\section{Preliminary}

WiFi-based positioning methods can be divided into two categories. One is based on distance measurement. It measures and locates distance by the relationship between certain information of radio electromagnetic wave and distance, and its classical positioning method is based on distance measurement. The method is trilateral positioning: the estimated coordinates are obtained by geometric method according to the distance between the positioning target and each reference point. In order to locate space and time in two-dimensional plane, only three points which are not on a straight line are selected as reference points. Firstly, the location information of the three points is known. After knowing the distance between them and the location target, the location of the location target can be calculated. 


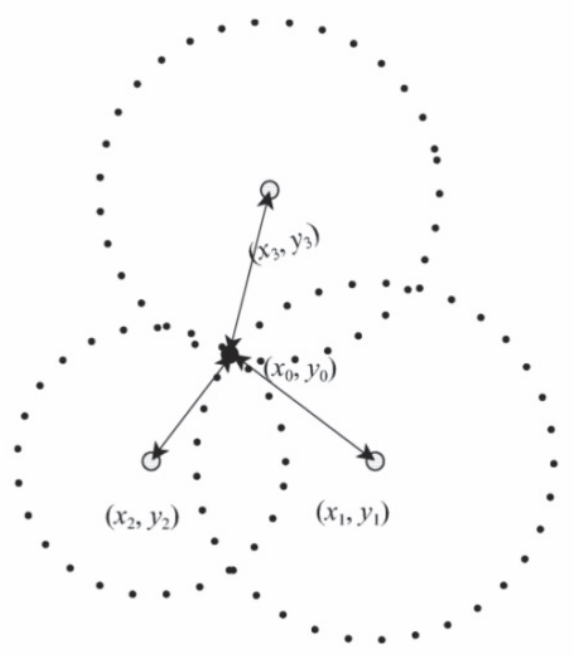

Figure 1. Three points localization method

As shown in Figure 1, the coordinates of the known reference nodes A, B and C are $\left(\mathrm{x}_{1}, \mathrm{y}_{1}\right),\left(\mathrm{x}_{2}, \mathrm{y}_{2}\right),\left(\mathrm{x}_{3}, \mathrm{y}_{3}\right)$, and their distances to the nodes to be located are $\mathrm{d} 1, \mathrm{D} 2$ and $\mathrm{d} 3$, respectively. If the coordinates of the points to be located are $(\mathrm{x} 0, \mathrm{y} 0)$, then

$$
\begin{aligned}
& \left(\mathrm{x}_{1}-\mathrm{x}_{0}\right)^{2}+\left(\mathrm{y}_{1}-\mathrm{y}_{0}\right)^{2}=\mathrm{d}_{1}{ }^{2} \\
& \left(\mathrm{x}_{2}-\mathrm{x}_{0}\right)^{2}+\left(\mathrm{y}_{2}-\mathrm{y}_{0}\right)^{2}=\mathrm{d}_{2}{ }^{2} \\
& \left(\mathrm{x}_{3}-\mathrm{x}_{0}\right)^{2}+\left(\mathrm{y}_{3}-\mathrm{y}_{0}\right)^{2}=\mathrm{d}_{3}{ }^{2}
\end{aligned}
$$

Then $\mathrm{x}_{0}$ and $\mathrm{y}_{0}$ can be calculated to achieve positioning.

RSSI fingerprint location method abstracts indoor physical location information into a RSSI signal intensity map of multiple AP using WiFi signal intensity information. Different RSSI signal intensity information represents different positions. The RSSI signal intensity information of multiple AP in all locations is stored in the database according to the corresponding relationship between location and RSSI, and a fingerprint database of RSSI signal intensity in all locations is obtained. In the positioning stage, by comparing the signal intensity information of the same AP in the fingerprint measured at the test point with the information stored in the fingerprint database, the signal intensity information in the fingerprint database is compared with the signal intensity information obtained from on-line measurement, and the identical or similar fingerprint is taken as the estimated location of the location.

\section{Method}

RADAR is the pioneering work of indoor positioning using WiFi fingerprints. The starting point of RADAR is that the RSS value of the same AP is relatively stable in space and has a certain degree of discrimination. For the WiFi signal generated by the same AP, the RSS value received at different locations will be significantly different. Based on the characteristics of these two RSS distributions, we call the WiFi signal fingerprint of a given location a high-dimensional vector consisting of RSS values of multiple signals arranged together, and locate unknown nodes by calculating the similarity between different fingerprints.

However, in fingerprint similarity comparison, RADAR uses all fingerprint records, which results in prolonged positioning time and affects positioning accuracy. Therefore, an adaptive fingerprint length selection method is proposed in this paper. The basic idea is to use different proportion of fingerprint length to make similarity comparison.

Specifically, it can be divided into two stages: off-line stage and on-line stage. In the offline stage, RADAR completes the construction of RSS-location database by collecting RSS spatial distribution information of all WiFi access points (AP).

In the online stage, the positioning system provides positioning services according to the constructed fingerprintlocation database. A node in an unknown location measures the RSS values of all AP in its current location and forms a query RSS fingerprint, which is uploaded to the location server. The location server calculates the similarity between the query fingerprint and the fingerprint in the database in turn, and returns the location result. 
In evaluating fingerprint similarity, the most commonly used method is to calculate the Euclidean distance between two fingerprint vectors.

\section{Experiments}

\subsection{Relation between RSSI Signal Intensity and Distance}

In the experimental environment, starting from the left side of the corridor, sampling is carried out at a sampling interval of 1 meter, moving along the straight line to the right, and the relationship between the received signal strength and distance is obtained as shown in Figure 2.
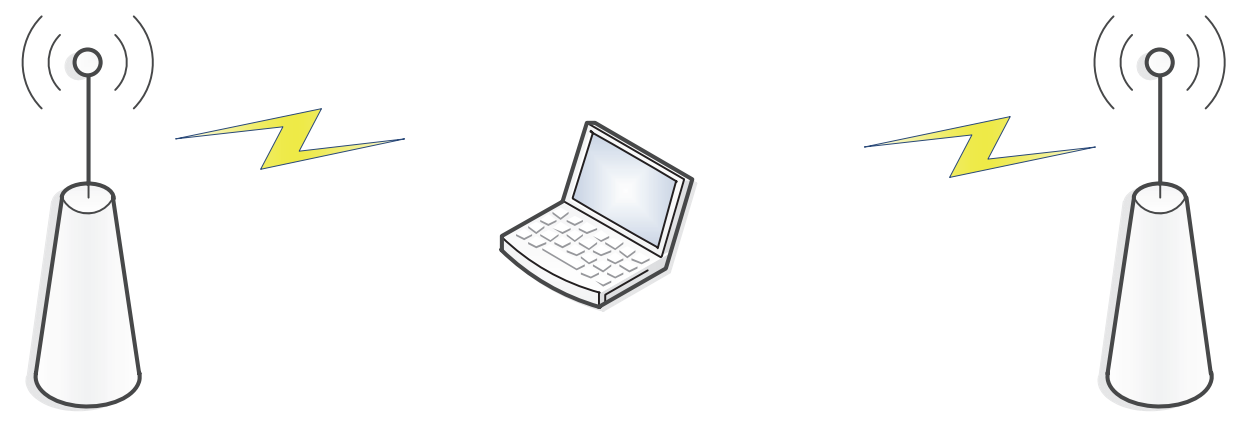

Figure 2. Expeiment Environment

The two curves are RSSI received from two AP in the figure 3 and figure 4. AP1 is on the left side of the corridor, AP2 is on the right side, and the X-axis represents the distance between the measuring point and the leftmost side of the corridor. As can be seen from the figure, the closer the terminal is to AP, the stronger the RSSI value received by the mobile terminal, and the lower the RSSI signal strength received when the terminal is away from AP. However, due to the interference of other factors in the indoor environment, the intensity between adjacent sampling points does not fully conform to the law, but fluctuates.

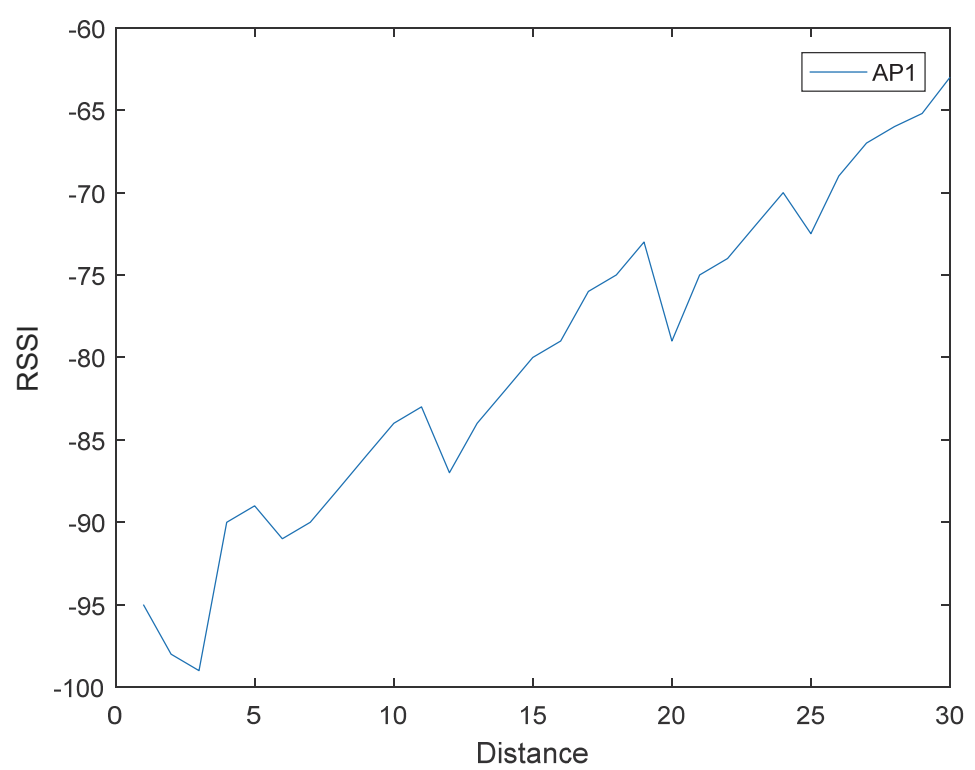

Figure 3. AP Value 


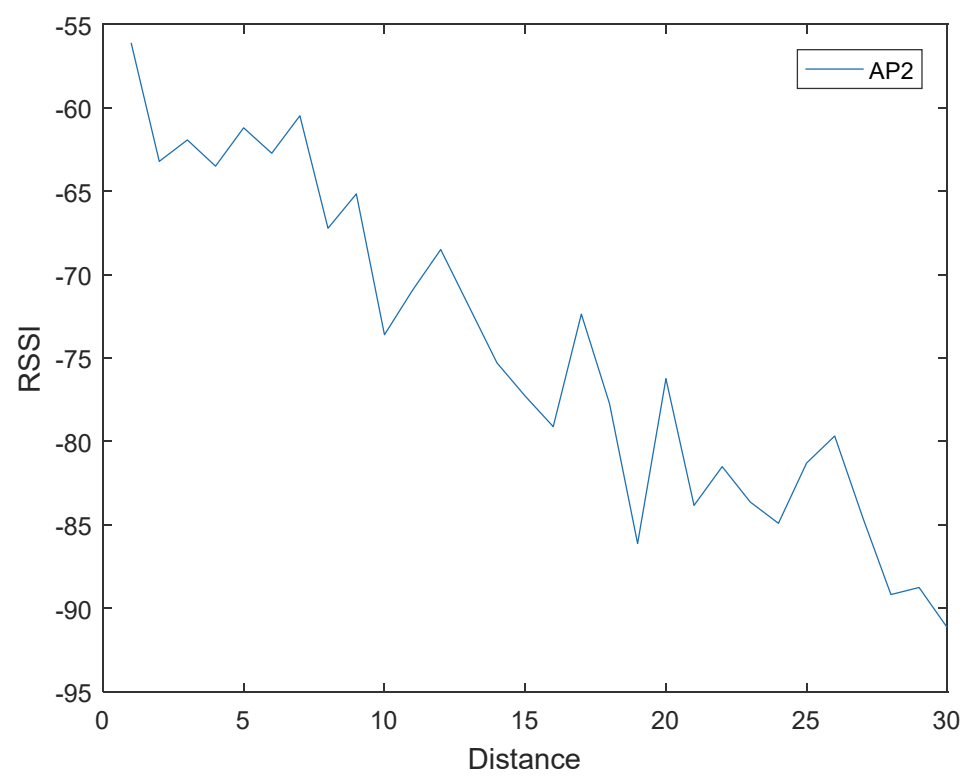

Figure 4. AP2 Value

According to the above research results, if the RSSI signal intensity difference between the locating point and a fingerprint point is large, the distance between them can be judged. This is the basis of discarding the fingerprint points with large difference in RSSI signal intensity when filtering fingerprint database. Next, we will study the RSSI fluctuation under different RSSI signal intensity mean, and provide experimental support for the size of filter interval.

\subsection{Our Method}

In this section, we show the performance of our method. The Figure 5 is the results.

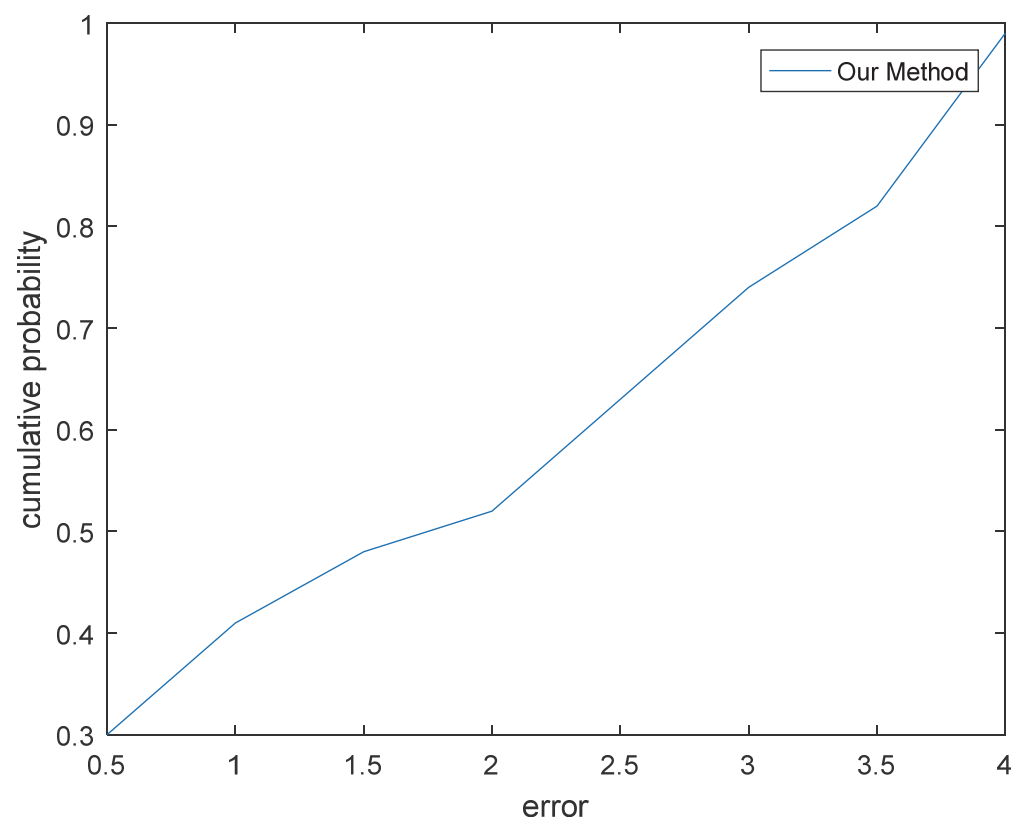

Figure 5. Result of our method

According to the above research results, if the RSSI signal intensity difference between the locating point and a 
fingerprint point is large, the distance between them can be judged. This is the basis of discarding the fingerprint points with large difference in RSSI signal intensity when filtering fingerprint database.

\section{Conclusions}

In this paper, through a lot of research on RSSI fingerprint positioning based on WiFi, we design an improved scheme for traditional positioning matching algorithm, and test the performance of the improved positioning algorithm through experiments. To a certain extent, the positioning accuracy is improved and the positioning delay is reduced.

\section{Acknowledgement}

This work is supported by National Social Science Foundation of China" Research on high intelligence value identification and job embeddedness feedback tracking model in big data Era" (No. 15BGL101), Six talent peaks project in Jiangsu Province"Research on high intelligence value identification and job embeddedness feedback tracking model in Internet + Era"(No. JY-030), and Young Academic Leaders Funded of Qing Lan Project of Jiangsu Province.

\section{References}

Au, W. S. A., Chen, F., \& Valaee, S., et al. (2013). Indoor tracking and navigation using received signal strength and compressive sensing on a mobile device [J]. Mobile Computing IEEE Transactions on, 12(10), 20502062.

Meneses, F., \& Moreira, A. (2012). Large scale movement analysis from WiFi based location data[C]. Indoor Positioning and Indoor Navigation (IPIN), 2012 International Conference on. IEEE, 2012, 1-9.

Milioris, D., Tzagkarakis, G., \& Papakonstantinou, A., et al. (2014). Low-dimensional signal-strength fingerprintbased positioning in wireless LANs [J]. Ad Hoc Networks, 12(1), 100-114.

Moghtadaiee, V., \& Dempster, A. G. (2012). Wi Fi fingerprinting signal strength error modeling for short distances [C]. Indoor Positioning and Indoor Navigation (IPIN), 2012 International Conference on. 2012, 1-6.

Niu, J., Lu, B., \& Cheng, L., et al. (2013). ZiLoc: Energy efficient WiFi fingerprint-based localization with lowpower radio[C]. 2013 IEEE Wireless Communications and Networking Conference (WCNC): SERVICES \& APPLICATIONS, 2013.

Yin, Z., Yin, Z., \& Yin, Z., et al. (2017). Gain Without Pain: Accurate WiFi-based Localization using Fingerprint Spatial Gradient [J]. Proceedings of the Acm on Interactive Mobile Wearable \& Ubiquitous Technologies, $1(2), 29$.

\section{Copyrights}

Copyright for this article is retained by the author(s), with first publication rights granted to the journal.

This is an open-access article distributed under the terms and conditions of the Creative Commons Attribution license (http://creativecommons.org/licenses/by/4.0/). 